\title{
ENDOSCOPIC APPROACH FOR LARYNGEAL AND HYPOPHARYNGEAL MALIGNANCIES WITH THE WEERDA DIVERTICULOSCOPE
}

\author{
Nikolay Sapundzhiev \\ Department of Otorhinolaryngology, \\ Medical University "Prof. P Stoyanov", Varna, Bulgaria
}

\section{SUMMARY:}

Introduction: The aim of the present work is to present our preliminary results for the use of the Weerda distending diverticuloscope (W DD) for diagnostic and surgical interventions in patients with upper airways and digestive system disorders, especially of malignant origin.

Material and methods: For the 2-years period 20082009 the W DD was used in 21 patients. Under general anesthesia and protection of the front teeth the W DD is introduced, following the course of the endotracheal tubus. By adjusting the blades of the instrument very good exposition of the whole larynx and partially of the hypopharynx are achieved.

Results: In 15 of the 21 cases the W DD allowed for optimal visualization of the region of interest. In 12/15 patients exploratory examinations with biopsies were performed. In 2/ 15 cases complete resection of carcinoma of the posterior pharyngeal wall was performed. In 1/15 cases arytenoidecomy was performed. In $6 / 21$ cases we were unable to introduce the instrument because of anatomical factors.

Conclusion: The W DD presents a versatile tool for better exposing the laryngeal and hypopharyngeal regions. It is a valuable complement to the standard surgical armament of the ENT H\&N surgeon, which gives new promissing diagnostic and treatment options.

Keywords: Weerda distending diverticuloscope, larynx, hypopharynx, squamous cell carcinoma

\section{INTRODUCTION}

Microscope enhanced endoscopy of the upper airways and digestive system (or panendoscopy) is an important step in the staging process of patients with head and neck $(\mathrm{H} \& \mathrm{~N})$ cancer. In general anesthesia all accessible mucosa of the upper airways and digestive system are examined to evaluate the tumor extension and biopsies are taken for histological diagnosis. In all ENT H\&N centers in Bulgaria this procedure is carried out with the means of the Klainsasser's type direct laryngoscope. We present our preliminary data on the use of another microsurgical system based on the new Weerda distending diverticuloscope [14].

\section{MATERIALAND METHODS}

In 2008 we introduced the use of a Weerda distending diverticuloscope (Karl Storz, Tuttlingen, Germany) in the routine protocol of staging patients with $\mathrm{H} \& \mathrm{~N}$ cancer (Fig. 1). The diagnostic procedure is carried out in a similar way with the standard Kleinsasser's technique. Under general anesthesia the patient's head is deflected. Under protection of the front teeth the Weerda diverticuloscope is gradually introduced, following the course of the endotracheal tubus. When the region of interest of the larynx is reached the diverticuloscope is fixed on the operating table with the standard support. By gradually turning the screw 1 (S1) the two blades of the device open at the top. This provides a very good exposition of the whole larynx and partially distends the hypopharynx. By gradually turning the screw 2 (S2) the proximal base of the blades opens and gives a larger access for the illumination, observation and the microsurgical instruments (Fig. 2). After examining the larynx, the blades are closed and the top of the instrument is moved to either pyriform sinus. Again the device is fixed and the blades are opened for examination/ manipulation. The procedure is repeated for the other pyriform sinus and for the retrocricoidal area.

\section{RESULTS}

Since the introduction of the Weerda distending diverticuloscope in 2008 at the Department of Otorhinolaryngology H\&N surgery it was used in a total of 21 surgical interventions. In 6 cases we were unable to introduce safely the instrument and changed it for the Kleinsasser's type of a smaller size. The reasons for unsuccessful use include: anatomically small mouth ( $2 \mathrm{x}$ women, $1 \mathrm{x}$ man), or limited mouth opening after radiotherapy in the head region $(1 \mathrm{x})$, dental factors (all superior teeth present or imminent injury of deteriorated superior teeth). In the 15 cases with successful introduction of the Weerda distending diverticuloscope a very good exposition of the larynx and hypopharynx were achieved (Fig. 3). In 12 patients exploratory examinations with biopsies we performed for staging and histological confirmation of malignancies of the upper airways and 
digestive system. The broad field offered by the instrument at its tip allowed for overview of the whole region of interest with very good delineation of the borders of the tumor mass. In one patient we were able to perform endoscopic arytenoidecomy for bilateral vocal fold paralysis. In further two cases complete resection of carcinoma of the posterior pharyngeal wall was performed (Fig. 4).

\section{DISCUSSION}

Since its introduction to the market in 1983 so far the Weerda diverticuloscope has been predominantly used for endoscopic treatment of Zenker's diverticulum [4], but also for other indications including lingual tonsillectomy [13], resection of hypopharyngeal diverticulum after laryngectomy [7], resection of esophageal polyps [6], laryngeal plastic [3], removal of pharyngeal foreign bodies $[5,12]$, endoscopic myotomy of the cricopharyngeal muscle [2]. We attempted the use of the Weerda diverticuloscope in the routine endoscopy for staging and biopsy of laryngeal and pharyngeal cancer. The most challenging cases operated with the help of this new instrument included 2 male patients with squamous cell carcinoma or the posterior hypopharyngeal wall. The posterior localization of the hypopharyngeal carcinoma is quite rare. It represents up to $10 \%$ of all cases of cancer of the hypopharynx $[1,11]$. The recommended treatment strategies include surgery (open lateral pharyngotomy, excision of the tumor and coverage of the defect with split skin) followed by radiotherapy. Another optional strategy includes radiotherapy, followed by meticulous follow up. With relatively small figures of patients being followed up with these diseases there is still no uniform opinion about the treatment algorithm [11]. For our patients we adopted the approach of transoral resection as in all 3 cases the primary tumor was T1-T2 with no radiological evidence of regional metastases. Using the Weerda distending diverticuloscope and cold-steel resection technique we removed completely the 2 tumors of the posterior pharyngeal wall in a way similar to the approach recommended by Rudert [8]. The treatment continued with radiotherapy and close follow-up as recommended by Sesterhenn et al. [10].

As estimated by our initial experience the major advantages of the new Weerda distending diverticuloscope include:
- It is a resizable instrument

- When the blades are opened at the tip the instrument provides a remarkably large overview at the area of interest. The visible field exceeds several times the field of the Kleinsasser's system

- The slits between the blades at the sides provide extra space for the surgical instruments and a better working angle

- The opening at the tip is undependable of the entry opening at the jaws

- Especially in the hypopharynx the new instrument provides soft but broad retraction of the soft tissues.

Some drawbacks of the Weerda distending diverticuloscope, especially in comparison with the Kleinsasser's direct laryngoscope, include:

- The handling of the instrument is more complicated and the operator should go an important learning curve because of the more complex handle and the adjustable screws

- It is difficult to fix the larynx with the Weerda diverticuloscope in order to perform safe extubation and intubation for intermittent apnea [9]

- When closing the blades in order to move the instrument to another area the blades could clamp the tracheal tubus - a risk for its dislocation or even extubation and loss of the airway.

\section{CONCLUSION}

The Weerda distending diverticuloscope presents an important aid in the diagnostic and therapeutic endoscopy of laryngeal and hypopharyngeal cancer. In the majority of cases it offers much better exposition of the spaces for examination, biopsy or resection. However it can not completely substitute the classical Kleinsasser's tools, because they still are more easily operated, allow for better fixation of the larynx and need less special experience to be located and relocated. The Weerda distending diverticuloscope is a valuable complement to the standard surgical armament of the ENT H\&N surgeon, which gives new diagnostic and treatment options for the endoscopic interventions in the laryngeal and hypopharyngeal regions. 

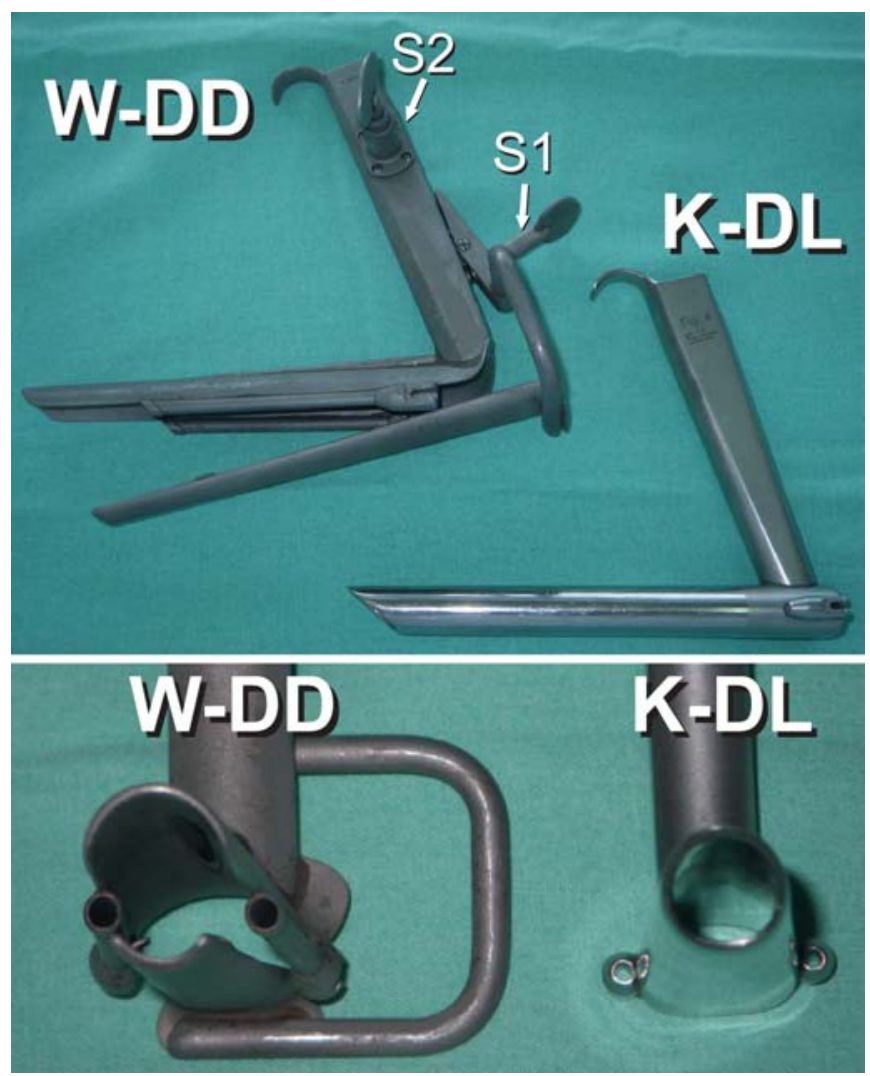

Figure 1: Weerda distending diverticuloscope (WDD) compared with the Kleinsasser's direct laryngoscope (K-DL). The screw-driven mechanics allow for opening of the blades only at the top (S1) and for opening the base of the blades at the patient's mouth (S2).

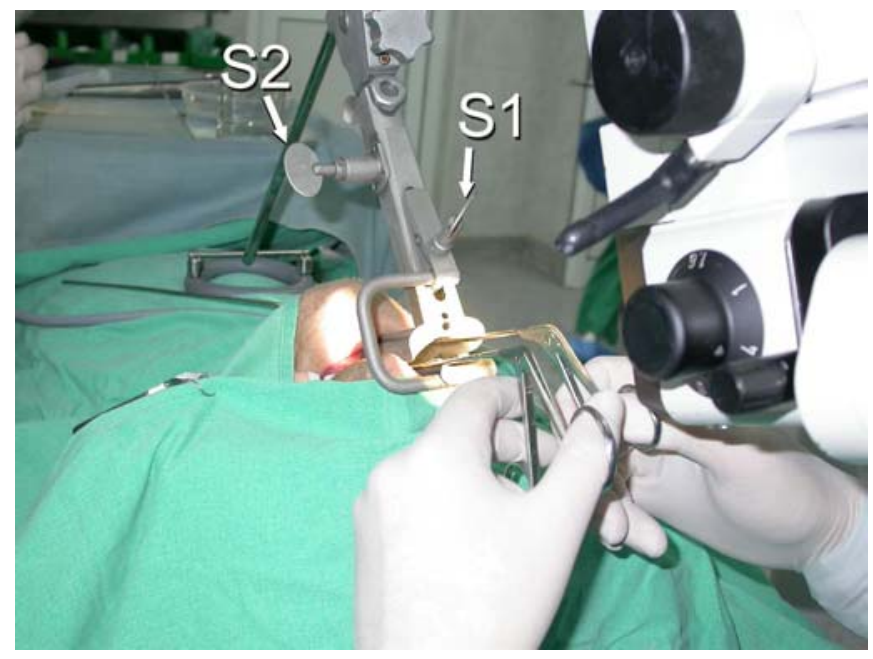

Figure 3: Endoscopic surgery of the larynx performed with the Weerda distending diverticuloscope (W$\mathrm{DD})$. Screws for opening the top (S1) and the base (S2) of the diverticuloscope.
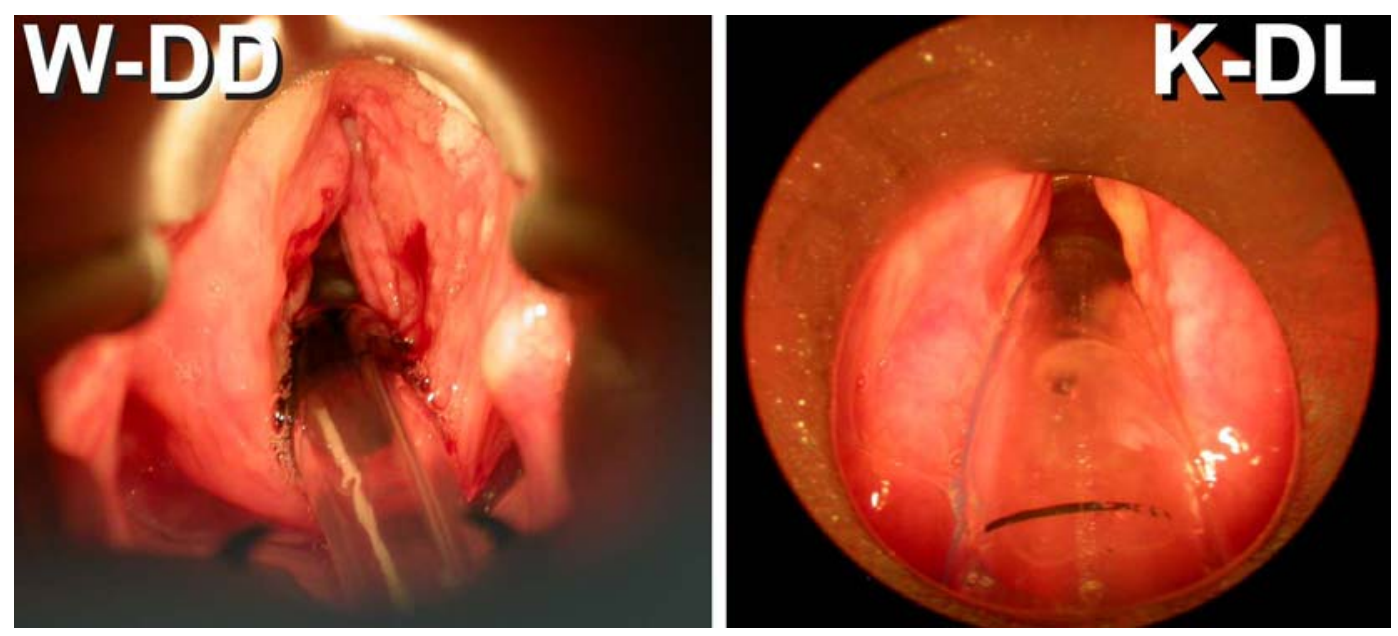

Figure 2: Surgical field at the larynx/hypopharynx as seen through the Weerda distending diverticuloscope (WDD, left hand side) and through the Kleinsasser's direct laryngoscope (K-DL, right hand side). 

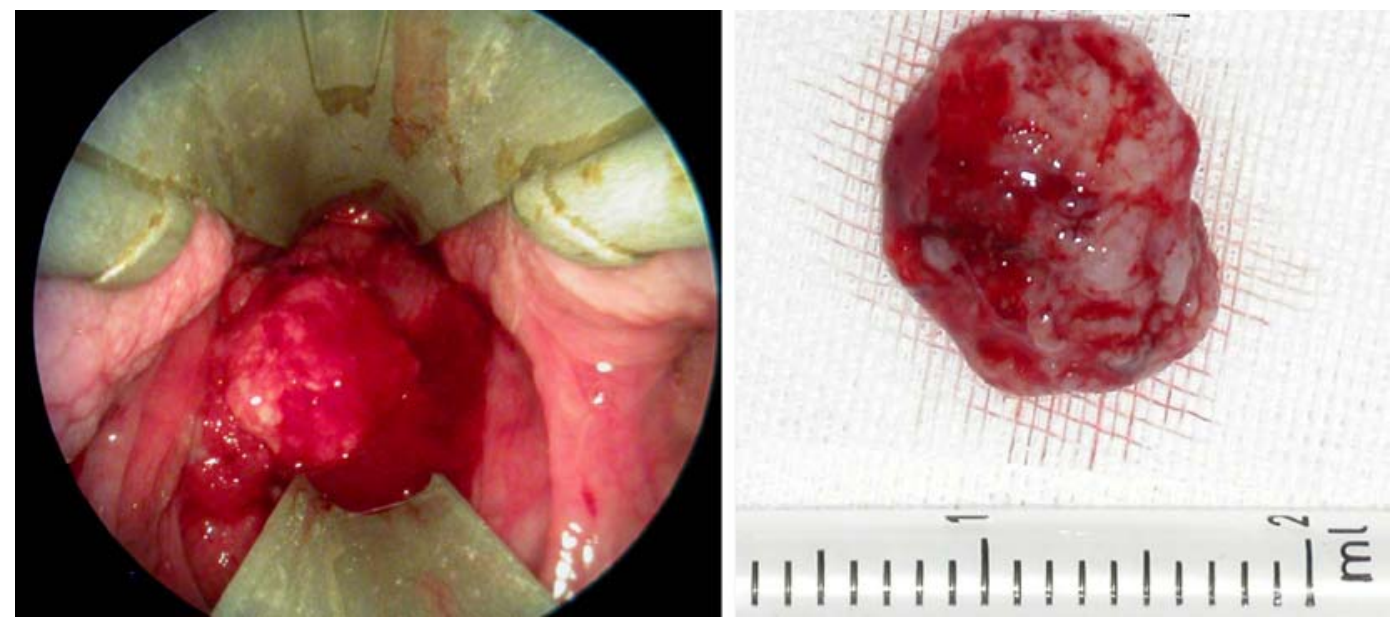

Figure 4: Resection of a T1 squamous cell carcinoma of the posterior hypophryngeal wall through the Weerda distending diverticuloscope (W-DD).

\section{REFERENCES:}

1. Arnold W, Ganzer U. Hypopharynxkarzinom. In Checkliste HNO-Heilkunde. 2005, Georg Thieme Verlag, Stuttgart. 406 408

2. Brwndbo K. Treatment of cricopharyngeal dysfunction by endoscopic laser myotomy. Acta Otolaryngol Suppl. 2000;543:222 224

3. Herrmann IF, Arce-Recio S. Special techniques for resolving aspiration problems. Operative Techniques in Otolaryngology 1998;9:180 191

4. Jaramillo MJ, McLay KA, McAteer D. Long-term clinico-radiological assessment of endoscopic stapling of pharyngeal pouch: a series of cases. J Laryngol Otol 2001;115:462-466

5. Kucharczuk JC, Kaiser LR, Marshall MB. Weerda diverticuloscope: novel use to remove embedded esophageal foreign bodies. Ann Thorac Surg 2003;76:1276 1278
6. McLean JN, DelGaudio JM. Endoscopic resection of a giant esophageal polyp: case report and review of the literature. Am J Otolaryngol 2007;28:115 117

7. Pitzer G, Oursin C, Wolfensberger M. Anteriores Pseudodivertikelnach Laryngektomie. HNO 1998;46:60 63

8. Rudert HH, Нцft S. Transoral carbon-dioxide laser resection of hypopharyngeal carcinoma Eur Arch Otorhinolaryngol 2003;260:198 206

9. Sapundzhiev N, Stelianova T, Gencheva M, Platikanov V. General endotracheal anesthesia with intermittent apnea technique in microlaryngeal surgery. Anaesthesiology and intensive care 2007;34:3 6

10. Sesterhenn AM, Muller HH, Wiegand S, Teymoortash A, Folz BJ, Werner JA. Cancer of the oro- and hypopharynx - when to expect recurrences?

Acta Otolaryngol 2008;128:925929

11. Stell PM, Bowdler DA. Chirurgie bцsartiger und gutartiger Erkrankungen des Hypopharynx. In Kopf- und HalsChirurgie in 3 Вдnden. 1998, Thieme Verlag, Stuttgart, NewYork. 189206

12. Swanson PB, Apicella SA, Rosen CA. Removal of a triple-barbed fishhook from the hypopharynx with microlaryngoscopy. Am J Otolaryngol 2002;23:233 236

13. Tucker Woodson B. Innovative technique for lingual tonsillectomy and midline posterior glossectomy for obstructive sleep apnea. Operative Techniques in Otolaryngology 2007;18:20 28

14. Weerda H, Pedersen P, Meuret G. A new distending laryngoscope for diagnosis and microsurgery of the larynx. Laryngoscope 1983;93:639 641

\section{Address for correspondence:}

Dr. Nikolay Sapundzhiev, MD

Department of Otorhinolaryngology, Medical University "Prof. P Stoyanov", 55, Marin Drinov Str., Varna 9002, Bulgaria e-mail: n.sapundzhiev@gmail.com 